\title{
Modelos de Flexibilidad del Trabajo y Tiempo Productivo: Estudio de Caso
}

\author{
Carlos Lozares * \\ Joan Miquel Verd **
}

RESUMEN:

El contenido del artículo se centra en la influencia que la organización flexible del tiempo en las empresas tiene sobre sus trabajadores y extensivamente sobre la sociedad. El objetivo del mismo consiste en identificar la manera en que la organización productiva del tiempo influye e interactía con el usos del tiempo en otros dominios de la vida social. Para ello se presentan tres modelos ideales de organización temporal contrastándolos con los datos empíricos que provienen de un caso de estudio. El caso elegido muestra grandes diferencias en la organización de la producción y del tiempo de trabajo en sus secciones. De esta manera en posible relacionar los diferentes perfiles de trabajadores con las diferencias en sus estrategias de negociación temporal y con su organización y percepciones del tiempo social.

\section{Palabras Claves:}

flexibilidad, modelos ideales, tiempo social, tiempo de trabajo, estrategias $y$ negociación de tiempo.

- QUIT (Grup d'Estudis Sociològics sobre la Vida Quotidiana i el Treball)

Profesor de Sociología. Universitat Autònoma de Barcelona - carlos.lozares@uab.es

* QUTT (Grup d'Estudis Sociolögics sobre la Vida Quotidiana i el Treball)

Profesor de Sociología. Universitat Autònoma de Barcelona • joanmiquel.verd@uab.es 
ABSTRACT:

The article focuses on the influence of companies' flexible organisation of time on their workers and extensively on society. The objective is to identify bow the productive organisation of time influences and interacts with the use of time in other domains of social life. For attaining this objective three ideal models of organisation of time are presented, which are compared with the empirical data coming from a case study. The case choosen shows big differences in the organisation of production and of working time in its sections. Thus it is possible relating the different profiles of workers with the differences in their strategies of time bargaining and with their organisation and perceptions of social time.

KEY WORDS:

flexibility, ideal models, social time, working time, stratagies and bargaining of time.

\section{INTRODUCCION}

El concepto de flexibilidad es decisivo para comprender la naturaleza y dinámica de algunos de los cambios que tienen lugar en el ámbito del trabajo productivo y, consecuente y extensivamente, en otros ámbitos sociales. La aplicación de cualquier nivel de flexibilidad en la empresa tiene evidentes consecuencias sobre la productividad, la contratación y los salarios, sobre las competencias, aptitudes y actitudes de los trabajadores, sobre el proceso y contenidos del puesto de trabajo, sobre la movilidad espacial y funcional, sobre los contratos y los salarios, y sobre la desestructuración de los tiempos de trabajo (cantidad, ritmos, cadencias, pausas) en unidades de medición flexibles (horas, días, semanas, años, tiempo de vida de trabajo). Pero no sólo afecta internamente al ámbito del trabajo productivo sino a la vida cotidiana de los/as trabajadores/as: inestabilidad del empleo, desestructuración de horarios y deslocalización de actividades y espacios, salud, derechos laborales adquiridos tan arduamente a lo largo de decenios. Nuestro punto de vista es que tal concepto ha de "asumir" e incorporar a su misma identificación las consecuencias que conlleva para los sujetos sociales involucrados y extenderse más allá del campo social de la producción.

El presente artículo aborda, precisamente, los efectos que comporta la flexibilidad productiva, y más concretamente la temporal, en la esfera de la empresa y en

1. Se trata de la investigación que denominamos Las implicaciones del reparto del trabajo sobre el empleo y la vida cotidiana llevada a cabo entre los años 1999 y 2001 en el seno del QUIT. La investigación fue financiada por la DIGICYT (SEC98-0570). Los objetivos de la investigación iban más allá de lo que aquíi presentaremos, y en ese sentido otro articulo de este mismo volumen recogen otros elementos relevantes. 
otros ámbitos de la vida cotidiana a partir de un estudio de caso. ${ }^{1}$ Para ello presentamos, en primer lugar, el concepto y tipos de flexibilidad productiva, en segundo lugar tres modelos ideales de organización productiva y temporal y más extensamente social, en tercer lugar, la comprobación, en los resultados, de la adecuación de dichos modelos ideales a la realidad de la empresa estudiada, y, en cuarto lugar, las conclusiones generales.

\section{La fleXibILIDAD DEL tRABajo y TIEMPO PRONUCTIVOS}

\subsection{LA fleXIBILIDAD, CONCEPTO CLAVE DEL PROCESO SOCIOPRODLICTIVO ACTUAL}

Desde la década de los 80 la idea de flexibilidad es recurrente en el discurso empresarial. Tomada de los planteamientos económicos neoclásicos, la idea de flexibilidad es sinónimo de desregulación: la supuesta esclerotización de la economía occidental -y especialmente en los países europeos- debe resolverse rompiendo con las rigideces institucionales que convierten el mercado de trabajo en un mercado "intervenido" y por lo tanto ineficiente (Nielsen, 1991). Este planteamiento hace especial incidencia en la flexibilidad numérica, aunque la teoría de la especialización flexible (Piore y Sabel, 1990) ha añadido nuevos elementos. Para estos últimos autores la organización productiva de las empresas debe ser más flexible para poder responder a una demanda que cada vez es más segmentada, diferenciada y cambiante, de modo que la producción se diversifique cuantitativamente y cualitativamente. De igual modo, la extensión de prácticas como la externalización o la producción ajustada (just in time) han terminado por imponer un nuevo paradigma organizativo y de racionalidad capitalista fundamentado en la empresa flexible (Kern y Schumann, 1989).

Tomando la clasificación de Brunhes (citado por Ruesga, 2002: 259), podemos distinguir entre flexibilización interna y externa, que a su vez se subdivide en diferentes subformas. Dentro de la flexibilidad externa, puede distinguirse entre flexibilidad cuantitativa, que afecta al número de empleados contratados (recurriendo a la utilización de despidos o la contratación temporal) y externalización (que recurre a las subcontratas y el prestamismo laboral). Dentro de la flexibilidad interna, se diferencia la flexibilidad cuantitativa, que modifica la cantidad total de trabajo utilizando con variaciones el tiempo efectivo de trabajo realizado, la flexibilidad funcional, que utiliza a los empleados en funciones variables según las necesidades de la empresa, y la flexibilidad salarial, que vincula los niveles retributivos de los trabajadores a la evolución de los resultados empresariales.

A lo largo del artículo abordamos principalmente los efectos que la flexibilidad cuantitativa interna tiene sobre los horarios y la organización temporal, por lo cual nos referiremos a ella como flexibilidad temporal. Como se ve, nos circunscribimos a 
un tipo de flexibilidad que se centra en la organización del trabajo, ${ }^{2}$ aunque desde luego no puede desvincularse de las otras formas de flexibilidad, y particularmente de la que afecta al mercado de trabajo y a la contratación. No obstante, es bueno recordar que como se ha apùntado ya en la introducción, los efectos de esta flexibilidad desbordan y van más allá del ámbito de la empresa para afectar al resto de tiempos de la vida cotidiana.

\subsection{LOS MODELOS DE ORGANIZACIÓN TEMPORAL, PRODUCTIVA Y SOCLAL}

El tiempo social es el signo e indicador más evidente y directo en el que se manifiesta y repercute la flexibilidad productiva. Actualmente, tanto en la industria como en los servicios se está desarrollando de manera progresiva la producción continuada sin delimitación temporal, de modo que pueda aprovecharse al máximo el capital fijo instalado. La producción de bienes y servicios se acerca cada vez más, por consiguiente, a una producción sin interrupción durante veinticuatro horas al día, los siete días de la semana. Se produce una desincronización evidente para aquellos/ as trabajadores/as que deben cubrir los turnos nocturnos o trabajar durante el fin de semana, puesto que la organización social del tiempo continúa desarrollándose en torno a unos horarios establecidos (Boulin, 1997).

El grado exacto de desincronización dependerá en buena medida de la organización temporal productiva y, tal como venimos señalando, del nivel de flexibilización temporal aplicado. Exponemos a continuación tres formas típicas ideales de organización temporal. La descripción de los tres modelos se va a centrar en torno a tres dimensiones básicas, que son también las que hemos considerado en el estudio de caso que presentamos: los aspectos relacionados con la organización temporal productiva; los relacionados con la organización temporal social, que incluye el resto de los ámbitos de la vida social; y los relacionados con la negociación y las maneras de aceptación y/o resistencia a una determinada organización temporal y horaria. Del encaje mutuo e interacción de los tres modelos citados surge lo que hemos llamado "organización temporal realmente existente" (véase la Figura 1), que a su vez es consecuencia de las relaciones de poder y de los condicionantes políticosociales que rodean a las personas. Los citados tipos de organización temporal, aunque sean (o hayan sido) hegemónicos en algún momento histórico, coexisten y se superponen en el momento actual, e incluso lo hacen en el interior de una misma organización productiva.

2. Se trata, sobre todo, de un modo de organización y gestión del tiempo productivo, y en ese sentidö va ligada al resto de elementos de la organización productiva en una empresa. 
Figura 1: Modelos que confluyen en la organización temporal de las personas

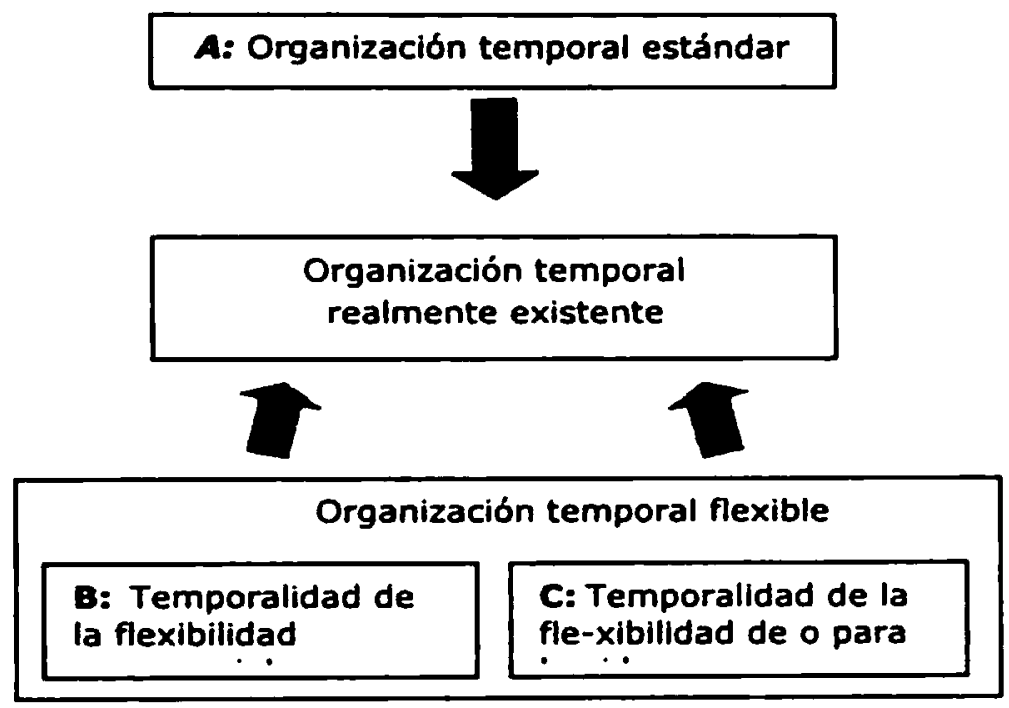

\section{- Modelo A. La organización temporal estándar}

Este modelo es una forma ideal de describir la forma clásica o estándar de organizar las actividades y tiempos del trabajo productivo propia a los inicios y, sobre todo, al apogeo de la sociedad industrial. Su ejemplo paradigmático se encuentra en el modo de producción taylorista, en que predomina una concepción del trabajo marcada por la planificación de su eficacia y la rentabilidad, y donde el tiempo juega un rol esencial en el sentido de que interviene tanto en la medición como en la planificación y ejecución eficaces. Su clímax llega cuando se traduce en cifras que miden la eficacia de los gestos y tareas realizadas. ${ }^{3}$ El tiempo se encuadra e inserta, como el trabajo, en un acuartelamiento cuasi-militarizado espacial e institucionalmente rígido y estable, y ambos se fragmentan al máximo para extraer las potencialidades instantáneas que da su continuidad, siendo además el/a trabajador/a un apéndice de la máquina que impone el imperio de sus temporalidades y movimientos. Para Foucault (1975) el sistema fabril usa diferentes procedimientos para capitalizar los tiempos de los individuos, acumularlos y organizarlos con provecho: (i) segmentar, es decir, dividir la duración en partes (por ejemplo, tiempo de aprendizaje, de formación, de ejecución, de inserción, de mantenimiento y de promoción); (ii) serializar los caminos y orientaciones tanto de las sucesiones simples como de las combinaciones complejas según un esquema analítico; (iii) limitar o poner cotas a las sucesiones temporales para

3. La cantidad de piezas producidas por unidad de tiempo será una especie de ley esculpida en mármol, y sobre todo la velocidad de ejecución, la precisión de gestos... Será al mismo tiempo lineal y repetitivo, con variaciones regulares en periodos largos, medios o infinitesimales. 
uniformizarlas y luego diferenciarlas en especialización de tareas; (iv) reconstruir que consiste en asumir la articulación de ejercicios coherentes y armoniosos en una especie de polifonía de actividades disciplinares. Todo ello conlleva y permite extraer provecho de la continuidad, evolución y de la sucesión. Además, con la segmentación y reconstrucción de la articulación, los tiempos de unos se ajustan a los tiempos de los otros de forma que se pueda extraer y combinar de manera óptima la mayor productividad. La articulación y su acumulación lineal producen la plusvalía y su dominación, la apropiación del tiempo por parte del capital. ${ }^{4}$

El tiempo, por lo tanto, es un componente básico en las sociedades industriales por varias razones: (i) por una fundamental que acabamos de señalar: mide el trabajo y en cierta manera fija el valor de la mercancía, (ii) mide también la rentabilidad y/o productividad global del trabajo, el manual, el mecánico y el informacional, (iii) fija la eficacia de la mano de obra: segmenta el trabajo en porciones temporales elementales para referenciar y eliminar los movimientos inútiles controlando la precisión en la ejecución y rentabilidad de los gestos permitiendo también su comparación entre los/ as trabajadores/as, y (iv) separa de manera tajante la "natural continuidad" de la vida, en particular los momentos del trabajo y de los del no trabajo.

Este último aspecto marca la segunda componente de la temporalidad de la organización industrial, su extensión o repercusión social en otros ámbitos que los productivos. En la sociedad industrial las actividades y el tiempo de los otros ámbitos de la vida social como el transporte, los servicios de empresas públicas o privadas, de las instituciones como la educación, la sanidad, la administración, etc., están marcados o siguen las mismas pautas que las del trabajo productivo. Además la afectación no se refiere sólo a la temporalidad de un día sino a otras como la semana, las vacaciones, el retiro, etc. Esta supeditación del tiempo social al clásico de la producción y de los tiempos de todas las actividades a los del sector productivo ha favorecido la misma la rentabilidad económica de la producción y ha socializado el resto de la vida social en actividades, hábitos y formas de vida. Ha sido también un tiempo funcional con relación a la familia nuclear (QUIT, 2000).

La tercera componente temporal se refiere a la negociación temporal. En este contexto el tiempo del trabajo estándar se negocia como paquetes también estándar: es decir por la reducción de las horas semanales. Esta norma temporal ha sido la lógica de la negociación empresarial, visión por lo demás muy asimilada también pọr

4. Sin componentes sico-analíticos y semiológicos, autores como Naville o Friedman hacen referencias a contenidos similares sobre el tiempo del trabajo en la época industrial. El hecho de que el trabajador solamente ejecute un fragmento del proceso de producción hace necesario que esta parcelación de tareas o trabajo en migas o migajas (particularidades, especializaciones, reparticiones, distribuciones, separaciones, afectaciones, alternancias, diferenciaciones, etc.) se combine, articule y oriente hacia la producción de un objeto (Sivadon y Fernandez-Zoìla, 1983). 
los sindicatos. Esta realidad descrita, aunque extendida, está cambiando notablemente imponiéndose cada vez más ciertas formas de flexibilidad temporal. Además la reducción de tiempo no es un problema primordial para los empresarios si hay otras compensaciones en la dirección de la flexibilidad productiva (Hörning et al., 1995).

\section{- Modelo B. La temporalidad de la flexibilidad empresarial}

Este modelo ideal no tiene aún un calco general en la realidad de la sociedad actual, sin embargo su irrupción parcial en el mundo de la empresa comienza a ser importante, $\mathrm{y}$, desde luego, representa un objetivo apetecible y perseguido por las empresas. Evidentemente, la metamorfosis interna del trabajo productivo y de su tiempo se hace desde dentro del modelo temporal estándar, es decir. del viejo orden temporal. Ya no se trata sólo de fragmentar, articular, coordinar y orientar tareas a un fin (proceso lineal) en el interior de la cadena productiva, en cadencias más o menos largas de presencia del trabajador en la empresa. Se trata de un tiempo pautado, según diferentes unidades temporales convenidas a la empresa, siendo el conjunto de la mano de obra lo que es objeto de apropiación por la empresa. Esta apropiación se extiende también a la articulación del trabajo entre los/as trabajadores/as y de ellos/as con los artefactos mecánicos, robotizados, informacionales y de cálculo; articulación que también se lleva a cabo a distancia, on line o just in time, con redes de empresas en red (Castells, 1997).

Este modelo supone la transformación y cambio sustantivo en la organización del trabajo. En la organización temporal estándar entraban formas atípicas, trabajo de noche, cortes y cambios de trabajo o trabajo a tiempo parcial. Sin embargo, con la emergencia del trabajo a 24 horas o con el desarrollo de la producción just in time se promueven y producen pautas diferentes que afectan a la naturaleza del trabajo normal. Para referirse a algunas de estas tendencias, Durand (2001) utiliza el concepto de flujo tenso a partir del cual pone de manifiesto que las tareas ejercidas por cada trabajador/a dependen mucho más del flujo y del conjunto del proceso que del puesto de trabajo específico que ocupa. Este autor pone de manifiesto que dentro de la lógica del flujo tenso se obtiene un incremento del trabajo no a través de la aceleración de los ritmos de trabajo real, sino a través de la densidad de los tiempos; esto es, principalmente a través de la reducción de la porosidad del tiempo. La plusvalía no se crea solamente sobre la asociación espacial y limitada temporalmente del conjunto de la mano de obra, cronológica y puntualmente reunida en el espacio de la empresa, sino que se genera globalmente sobre la totalidad de una mano de obra y una tecnología, internalizada o externalizada a la empresa (o red de empresas), que está disponible "full time" y "on line".

Los cambios van mucho mas allá de una simple variación del tiempo clásico de las 40 horas semanales y del horario partido; es más extenso, profundo y complejo. El dominio de su tiempo se le escapa al/a trabajador/a ya que se extiende más allá de 
los límites de su actividad, tarea o función material en la empresa. Si a esto se añade el hecho de que el/a trabajador/a se encuentra cada vez más, debido a los artefactos manejados y en parte exigidos por la flexibilidad y la coordinación necesaria, con uno tipo de comunicación y Tenguaje de signos que supera al habitual de los gestos simples y mecánicos del lenguaje ordinario de la industrialización, se llega a la conclusión de que el trabajo productivo requiere más habilidad, polivalencia y flexibilidad funcional. Por otra parte, la mayor elasticidad y plasticidad en el trabajo con artefactos y en equipo provoca que el tiempo productivo, además de extenderse a todo el día, semana... sea instantáneamente más complejo y condensado. ${ }^{5}$

Dentro de las consecuencias sociales de este tipo de temporalidad propia de la organización del trabajo, Harvey (1999a,1999b) señala las dimensiones que marcan las diferencias entre el tiempo productivo estándar y el nuevo tiempo productivo: (i) primero, las horas de trabajo sociales y no sociales, es decir, las horas relacionadas con un trabajo asalariado, como resultado de unas relaciones de producción, por un lado, y, por otro, las horas no asalariadas; (ii) segundo, entre el tiempo de trabajo normal, atípico y el de notrabajo; (iii) tercero, las relaciones entre trabajo, moneda y tiempo. En definitiva, en el nuevo día de trabajo que alumbra la flexibilidad empresarial, el trabajo diario está lejos de ser una simple suma de 8 horas y la organización del tiempo productivo de corresponder a sumas homogéneas de una cronometría plana; más bien se realiza en superposiciones de duraciones, ciclos y sincronías diferenciadas. Se asiste más bien a un conjunto de intercambios en la compraventa de temporalidades diversas entre empresario y trabajador. La diferencia estriba en que lo que fue atípico ahora es normal pero no sólo en trabajos de noche, sino también en los nuevos contratos por horas, el trabajo a distancia, el trabajo "en casa", todas las formas temporales de contratación, las horas anualizadas, mensualizadas... Todo ello modifica los estándares de una concepción del tiempo productivo y las anteriores pautas, incluso las que denominábamos atípicas (Harvey, 1999b).

Un modelo como el descrito, llevado a sus últimas consecuencias, aboca a la destrucción del tejido social, al menos tal y como ha estado tramado hasta el presente y toca de fondo el tipo de organización social clásica del tiempo y del resto de la vida social. Y ello debido a la diversificación, multiplicidad, extensión y coexistencia de pautas temporales productivas en horarios, ritmos y duraciones diferentes, aparte del aislamiento y desajuste de las personas. La vida entera social (día a día, semana a semana, etc.) de los/as trabajadores/as y de las familias deberá estar pendiente y es dependiente de una laberíntica conjugación horaria en la empresa y de un juego imposible de simultaneidades, dislocaciones y fragmentaciones de temporalidades en

5. Si aún queda algún resquicio en la vida ya absorbida del trabajador (a disponibilidad continua) este espacio es recuperado y "rescatado" para el sistema empresarial por la lógica del consumo dirigido a partir, precisamente (ironías de la plusvalia que se cierra sobre sí misma), de los productos que él mismo ha fabricado; la lógica fordista subsiste para los tiempos de ocio y de consumo. 
el seno de los grupos primarios como familia o amistades y de los secundarios (Sivadon y Fernandez-Zoïla, 1983 ; Boulin, 1992, Lallemant, 2003).

En relación con la tercer aspecto considerado, la negociación y aceptación de estos cambios, frecuentemente, la posición de los representantes de los/as trabajadores/as les lleva a enfrentamientos con determinados grupos de trabajadores/as sea porque éstos no aceptan ningún tipo de flexibilidad por lo que supone de desestructuración de sus tiempos sociales, sea porque la única negociación que desean es la de las horas extras que puede conllevar, por el contrario, a no conseguir otras contrapartidas de la negociación como son las de nuevas contrataciones o más empleo estable (Martín Artiles, 1995: 252-254).

A juzgar por el desarrollo de muchos pactos y negociaciones, los tiempos y actividades del trabajo, así como la misma organización productiva, comienzan a tomar un cariz flexibilizador de efectos desincronizadores y deslocalización espacial y social. El interés por un tiempo productivo absolutamente flexible por parte del empresario, aparte de otras razones ya anunciadas, proviene esencialmente del hecho de que, por ejemplo, un tercio más de horas de trabajo diario (o semanal, mensual, anual o de vida laboral) organizado de forma flexible no es equivalente a un tercio más de horas de trabajo organizado de forma estándar, como tampoco lo es para el/la trabajador/ a un tercio menos de tiempo libre en "tiempo estándar" que en "tiempo flexible".

\section{- Modelo C. La temporalidad de la flexibilidad de o para la vida}

Este modelo, también ideal -aquí en un doble sentido de tipo ideal y objeto de deseo -, se presenta en clara ruptura, por un lado, con el modelo clásico propio de la sociedad industrial y de su tiempo estándar $y$, por otro, con el de la flexibilidad empresarial. Como señala Recio (1997a: 161-162), generalmente la flexibilidad se plantea como vía para que los/as asalariados/as se adapten a las necesidades de las empresas y en muy raras ocasiones se considera que la flexibilidad pueda plantearse en una dirección inversa. En estos casos, cuando las demandas de flexibilidad se realizan para satisfacer las necesidades particulares de las personas, suelen ir orientadas a la adaptación de la jornada laboral a las necesidades vitales, como tiempo para el estudio o para cuidar a otras personas. Otra de las demandas de cambio en el tiempo productivo -reducción, en este caso- que no surge del lado empresarial se plantea en las discusiones sobre el reparto del empleo. De todos modos, tal como señala Recio (1997b), para enfrentarse a los cambios que se producen, y más concretamente al desempleo, no se puede pensar en el reparto del empleo a partir de la reducción del tiempo de trabajo según la lógica del tipo hegemónico de sociedad. Un tal planteamiento se hace sin tener en cuenta que la reducción del tiempo de trabajo debe suponer ante todo un cambio del modelo de sociedad porque necesariamente ha de tocar a la redistribución de la renta. 
Otra aproximación a este modelo de "flexibilidad de y para la vida", de carácter radical e ideal, plantea la hegemonía en el mundo social de un tipo de flexibilidad en que las actividades y tiempos sociales sean autogestionados a la medida de la persona y de todos sus contextos dé referencia o pertenencia. Este modelo aboga o sostiene el declive paulatino del trabajo productivo en volumen, dedicación y peso social con la correspondiente superación del sistema social que lo mantiene y del orden, legitimación y valores que le corresponden. De alguna manera significa entonces que cualquier camino que se emprenda en la dirección de disminuir el paro aumentando la masa de producción está llevado al fracaso porque la tendencia opuesta es inexorable. $Y$ que cualquier intento de redistribución del volumen de empleo y trabajo productivos realmente existentes significará un reparto cada vez más raquítico y menguado de un bien, a su vez, cada vez más escaso. Es decir, que se trata del planteamiento de otras lógicas y estrategias y de otras formas de organización social como alternativas al sistema de producción vigente. Según los patrocinadores intelectuales de este planteamiento, el advenimiento de otro tipo de sociedad no vinculada al empleo como forma hegemónica de desarrollo y estructuración es inexorable (Gorz, 1995, 1997).

Los tiempos productivos son, bajo esta aproximación, cada vez más reducidos aunque de naturaleza obligada, los tiempos autogestionados por las personas y por las colectividades son cada vez más frecuentes, amplios y hegemónicos: se trataría de los tiempos de educación no reglada, formación permanente y continuada, de distribución del trabajo doméstico, del cuidado de las personas y de los mayores y de los servicios personales, de las actividades sostenibles, etc. Parece natural que estas tesis sean coherentes con quienes hablan de un cambio profundo de sociedad proveniente de las rupturas radicales que se dan en el ámbito de la producción (Gorz, 1999: 103-110; Sue, 1982, 1994).

La aproximación de Zoll $(1988,1994)$, es menos radical en el sentido de que la reducción del tiempo de trabajo es necesaria pero no suficiente para eliminar el paro aunque con los cambios habidos en la innovación tecnológica y en la organización productiva se necesitan cada vez menos puestos de trabajo. En consecuencia, el crecimiento económico y la inversión no suponen necesariamente más puestos de trabajo. La solución al paro pasa por una reordenación de los tiempos de trabajo y de no trabajo, y por la creación de un segundo sector en servicios sociales, ecológicos y culturales. Dicho sector, no mercantilizado, sería alternativo y cohabitaría con el mercantilizado; favorecería el desarrollo de la conciencia cívica frente a la conciencia mercantil, estaría organizado con criterios de reciprocidad según una renta ciudadana y estaría sostenido por el sector mercantil. Supondría la aparición de un tiempo "reproductivo social" que abarcaría espacios sociales más amplios que los del trabajo reproductivo o doméstico-familiar actual. 
3. LOS RESULTADOS DEL ESTUDIO DE CASO: FLEXIBLIDAD Y ORGANIZACIÓN PRODUCTTVA, CONSECUENCLAS SOCIALES Y NEGOCIACION.

Veamos, en este apartado, algunos de los resultados directos del estudio de caso llevado a caso en una empresa y sobre un colectivo concreto de trabajadores y trabajadoras de la misma. Las técnicas utilizadas en la investigación fueron principalmente tres: una encuesta realizada a 400 trabajadores de la empresa, 6 grupos de discusión con trabajadores/as diferenciados por género y sección, y entrevistas informativas a directivos y sindicalistas y en profundidad a trabajadores/as de la empresa. En estos resultados se van intercalando tanto los que provienen de datos cuantitativos como los de datos cualitativos. Seguimos lo más cerca posible las pautas que han guiado la descripción de los modelos: flexibilidad horaria y organización productiva; los efectos de la flexibilidad horaria sobre otros ámbitos de la vida social a través de las representaciones que se hacen los/as trabajadores/as; y las cuestiones vinculadas a la estrategias y negociación de los tiempos.

El caso estudiado es el de la filial española de una empresa multinacional de capital japonés radicada en la comarca del Vallés Occidental (Barcelona). Los principales productos que fabrica la planta española son televisores y videos, junto con algunos otros componentes que se venden sin ensamblar. La demanda de los productos que fabrica tiene oscilaciones durante el año, a las que la empresa responde mediante una producción just in time, aumentando o disminuyendo el nuimero de lineas de producción en servicio y aumentando o disminuyendo el número de operarios por linea (mediante la contratación temporal).

3.1. La tipología de horarios y la organización PRODlictiva de las SECciones de la EMPRESA.

El cuadro que viene a continuación resume según tipos diversos algunas de los elementos que definen la sectorialización y diversificación de los sistemas horarios en la empresa estudiada.

Cuadro 1: Tipología de sistemas horarios en la empresa estudiada

\begin{tabular}{llll} 
Tipo 1 & Tipo 2 & Tipo 3 & Tipo 4 \\
\hline
\end{tabular}

\begin{tabular}{|lllll|}
\hline Sección: & de Inserción & de Producción & de Producción & de Diseño \\
\hline Horario: & Turnos rotatorios & Turno de mañana & Turno de tarde & Partido \\
\hline $\begin{array}{l}\text { Horas de } \\
\text { trabajo: }\end{array}$ & 40 horas o menos & 40 horas o menos & 40 horas & Más de 40 \\
\hline
\end{tabular}


El Tipo 1 corresponde a la sección de Inserción, que marca de alguna manera el volumen y la dinámica de la producción. Por tanto es donde más directamente se aplican las condiciones de la producción just in time. Por otro lado es donde más ha invertido la empresa en automatización y donde hay mayor necesidad de amortización. Todo ello hace que sea "el lugar" donde mayor implantación tiene la flexibilidad temporal, en concreto con el trabajo por turnos rotatorios las 24 horas del día durante los 7 días de la semana. Esta flexibilidad extrema en el horario de trabajo se manifiesta por la organización rotativa del trabajo en turnos quincenales, así como por los cambios de planificación en la distribución anual de los mismos en función de las necesidades de la empresa -se establece un quinto turno en las épocas de mayor demanda. El horario anual de la sección se organiza de forma tal que al inicio de año. se suministra un primer esquema de horario a los trabajadores, que después suele ser modificado en el mes de mayo.

Los Tipos 2 y 3 corresponden a las secciones de Producción, en que prima como horario y sistema de trabajo el modelo más tradicional de la producción taylorista: cadena serializada, manual y rígida. Existen dos turnos de horario continuado, uno de mañana y uno de tarde; no existe ni rotación ni se trabaja durante los fines de semana. De hecho es la sección y horario que más facilita la compatibilidad del trabajo en la empresa con otros tipos de dedicación, por ejemplo el trabajo doméstico-familiar. Por causas debidas al horario y otras que iremos completando es importante diferenciar los dos tipos de horarios señalados y las personas que trabajan por la mañana de las que trabajan por la tarde. Hay que indicar, de todos modos, que su forma "tradicional" es engañosa en el sentido de que en los períodos de fuerte demanda se incorporan a estas secciones trabajadores/as con contratos temporales de estación y definidos en horas anuales; es la manera de aplicar sobre una base del tipo de producción estándar propio a un modelo clásico una flexibilidad externa de tipo empresarial.

El Tipo 4 corresponde a la sección de Diseño, que es la unidad de creación y desarrollo tecnológico de la empresa, y donde se trabaja en equipo. Se trabajan 8 horas diarias en un sistema de horario partido pero pueden ser ampliadas diaria, semanalmente e incluso en vacaciones. Se trata de una flexibilidad temporal elástica y extensiva, muy determinada por las exigencias de la empresa, aunque normalmente no se trabaja durante el fin de semana. Es también la sección donde se trabaja con instrumentos más informatizados e inteligentes. Esta sección tiene un modelo horario clâsico aunque con condiciones que pudieran favorecer la aparición de alguna posibilidad de autogestión. Pero éste no es precisamente el caso, la capacidad de gestionar el propio tiempo se usa precisamente para alargar la jornada mediante horas extraordinarias. Así pues la característica más específica del tiempo de trabajo productivo en esta sección es la extensión y ampliación del mismo. El tiempo de trabajo invade el resto de los ámbitos de la vida cotidiana de los trabajadores, la semana y el año, tomando a veces de las vacaciones una semana de trabajo. 
Como se comprueba, la sección en la que se trabaja determina y contextualiza el sistema de horarios. Cada sección en la empresa conlleva un tipo y contenido de trabajo, un nivel de tecnificación y por ende un tipo de organización temporal concreta. La razón de esta variabilidad en la forma y modulación de la organización temporal dentro de la empresa se debe a causas diversas: la organización productiva de una sección específica, la tecnología desarrollada en ella y su amortización, la cualificación del personal, los flujos estacionarios de producción. La secciones no solamente condicionan el sistema de horarios sino que también se corresponden con formas de organización productiva diferentes $y$, por tanto, con tipos, contenidos y exigencias de trabajo diversos. Es una clara evidencia de que bajo una organización general flexible en la empresa subsisten secciones o departamentos que se corresponden con formas más clásicas de organización.

Todo ello se da, de todos modos, bajo el marco de una organización productiva general en la empresa que busca la eliminación de estocks, una producción just in time y un seguimiento muy atento de las fluctuaciones de demanda en el mercado. Puede afirmarse que la lógica global de la empresa sigue en algún grado la flexibilidad empresarial.

\subsection{FleXIBILIDAD, vivenCIA Y CONCIENCLA DEL TIEMPO SOCIAL}

Para las mujeres que trabajan en la sección de Producción, donde tienen una presencia considerable ( $y$ en particular en el turno de mañana, en que predominan las que viven con su pareja e hijos), el tiempo social es vivido de forma dual, trabajo y hogar, y la centralidad doméstico-familiar es mayor que en cualquier otra sección. El trabajo productivo y su tiempo no tiene para ellas una identidad autónoma, es visto por estas mujeres como una necesidad subsidiaria y ello tanto más si se vive en pareja, si se tienen hijos y si se es más mayor. No es este tanto el caso de las mujeres jóvenes.

En Inserción, la vivencia y realidad del tiempo son completamente asincrónicas -cronológicamente hablando- con los ciclos "socio-naturales" de vida, lo que supone una vida organizada en torno a la centralidad productiva. Las horas diarias de trabajo son cualitativamente mucho más que las 7 u 8 que trabajan. La sensación que se tiene es la de una delimitación borrosa e incierta de los diferentes tiempos dentro de un flujo y fronteras que además se desplazan y corren constantemente.

En Diseño la centralidad en el trabajo es completa y absorbente sin ser acíclica o variable. Vistas así las cosas es difícil concebir o tener una conciencia autónoma del tiempo social ajena a la imperiosidad que proviene de los tipos de centralidad productiva y menos de un tiempo autogestionado tal que la gestión del tiempo sea un valor central de una posible realización personal y colectiva. 


\subsection{LOS CAMINOS DE LA NEGOCIACIÓN}

Los términos de la negociación se plantean en tanto que objetivos generales en los términos siguientes. Primero, la estrategia de la empresa va encaminada al uso extensivo, continuo e intensivo de los recursos organizativo-productivos, tecnológicos y de la fuerza del trabajo, sobre todo del tiempo de vida del/a trabajador/a en vistas a la competitividad y a las demandas de un mercado variable, diferenciado y global. Por ello, la estrategia de la empresa va en la dirección de una flexibilidad generalizada. Segundo, el objetivo de los/as trabajadores/as está en la empresa, y ha estado en la historia, destinado a la reducción horaria -siempre que sea sin reducción de salario- y a las posibilidades de una mayor contratación estable en el empleo.

Sin dejar estas finalidades generales de lado como horizonte permanente de negociación entre intereses opuestos, en las condiciones reales los objetivos son más concretos y posibilistas. Lo que se "pone en la mesa" de negociación por ambas partes se centra más en la computación, distribución y redistribución de las horas según diferentes temporalidades, diarias o incluso en horas semanales y/o anuales; en los distintos tipos de horarios y turnos de trabajo; en las horas suplementarias; y en el aumento de la productividad, como alternativa a la flexibilidad temporal radical empresarial, por un lado, y, por otro, reducciones limitadas del tiempo global de trabajo, nuevas contrataciones y empleo estable y aumento salarial como alternativa a las exigencias maximalistas de los trabajadores/as.

Una reducción relativamente importante de tiempo de trabajo con reducción correspondiente de ingresos no es ni de actualidad ni de interés por parte de los/as trabajadores/as. Pudiera tenerlo si como contrapartida se mantuvieran los ingresos y siempre en vistas a aumentar el empleo y/o estabilizar a parte de la plantilla.

\section{ConClusiones}

Con la reticencia que impone el estudio de un caso, es decir, con una generalización prudente y contrastada con otras investigaciones y referencias que hemos ido introduciendo a los largo del artículo se pueden proponer como conclusión algunas líneas más generales referidas a cómo parece irse configurando la realidad actual.

Tomando como referente los modelos ideales propuestos, se puede suponer con bastante verosimilitud que la situación actual cabalga a trancas y barrancas entre las vicisitudes y la complejidad de la presencia e incidencia en ella de los tres modelos descritos con dosis más o menos ponderadas de cada uno según situaciones y condiciones. Parece que estamos en situación de interfase entre las reminiscencias muy presentes aún del primer modelo de la sociedad del empleo y del trabajo y del "tiempo estándar", de las incidencias cada vez más incisivas del segundo modelo de las exigencias productivas y sociales de la "flexibilidad empresarial", y de los utópicos deseos y proyectos que provienen de la "flexibilidad de y para la vida". En su conjun- 
to la vida social de los individuos, familias y grupos parece haber entrado en una larga fase de desajustes y acomodaciones, de incertidumbre y acoplamiento a las determinaciones y exigencias variables de los tres modelos que operan en simultáneo. Los individuos, las familias, en la empresas y en las colectividades, están obligados, y obligándose, a un continuo proceso de negociación múltiple y graduada entre sí y entre las exigencias que provienen de los diferentes ámbitos de vida, productivo, reproductivo y el de las actividades y tiempo del no-trabajo. En este sentido la imagen de una realidad como la actual es la de un cambio complejo e incierto en su solución pero en el que la salida más equilibrada a la problemática tratada ha de pasar por la negociación cotidiana y permanente en la empresa, en el hogar y entre hombres y mujeres.

Hay que pensar que el tiempo de trabajo influye en la vida extralaboral, que el trabajo también existe fuera del productivo, que la carga total del trabajo está desigualmente distribuida en perjuicio de la mujer, que el problema no es sólo el de tener más tiempo sino de que sea más libre y en qué, cómo, cuándo y para qué se emplea, que la "flexibilidad empresarial", panacea anunciada como imprescindible para el progreso económico, tiene consecuencias graves en la empresa y fuera de ella y que en el cálculo de lo que supone la "flexibilidad empresarial" se habrian de incluir también los gastos sociales, por ejemplo en convivencia, coherencia social o desigualdad de género (Recio, 1997b; Torns, 1998)

Lo que mejor puede identificar una realidad como la actual de contradicciones y complejidad, y por aquí va una hipótesis aceptablemente comprobada, es la idea de transición y de concomitancias de fenómenos simultáneos que se reflejan en diferentes formas de horarios, ritmos, cadencias y cortes en el trabajo, si nos atenemos sólo al mundo productivo. Pero hay que seguir afirmando, pese a los cambios, el hecho aún básico de que un día de trabajo sigue siendo trabajo de un día en producción, ( $\sin$ tener en cuenta el trabajo reproductivo), que la reducción temporal del trabajo productivo es aún menguado y que desde la empresa la reducción del tiempo de trabajo no es concebible sin el aumento de la productividad. Ello hace que el paso a la producción continua y fluctuante (y según el contenido y los ritmos de la demanda que está a la base de la exigencias de la productividad), se haga bajo múltiples regímenes temporales y de formas de contratación.

La organización temporal "realmente existente" es el resultado de efectos confrontados que provienen de exigencias e instancias diferentes: (i) las de la flexibilidad productiva como estrategia buscada declarada e insistente por parte de las empresas; (ii) las que proceden de inercias y hábitos horarios, sin connotación negativa alguna, de los/as trabajadores/as que son difíciles de cambiar por razones obvias; (iii) las que tienen su origen en determinadas condiciones familiares u otras; (iv) las que provienen de una mayor conciencia y necesidad de un tiempo de vida para sí, aunque su influencia se sitúa en el nivel de los desideratas casi nunca alcanzados. 
El cambio de las situaciones temporales en las empresas debido a la implantación de la flexibilidad empresarial puede modificar considerablemente la vida de los/as trabajadores/as acostumbrados/as con ritmos y necesidades temporales legítimos según sus intereses individuales-y familiares. De este modo, el empleo comienza a tener unas condiciones y connotaciones temporales (ritmos, cadencias, dependencias, periodos, rupturas, segmentaciones, articulaciones, etc.), es decir, unas temporalidades, que tienen poco que ver con la idea de trabajo vinculado a un puesto de trabajo o al tiempo de trabajo estándar. La situación social actual es la de connivencia o convivencia más o menos parcial y tensa de los modelos descritos. Precisamente su simultaneidad y superposición hace que la situación actual sea contemplada y vivida por las gentes en condiciones de incertidumbre, dualidad o inestabilidad que puede traducirse en formas conflictivas más o menos latentes. ${ }^{6}$

No es previsible que los/as trabajadores/as vivan en un modelo alternativo. Más bien se encuentran entre las cuerdas de un ring triangular: las reminiscentes de un modelo clásico en vigencia, las exigencias que provienen de las nuevas formas de organización del trabajo y del tiempo que adquieren unas cotas y cuotas cada vez más importantes y tensas y, las aspiraciones insoslayables a unos tiempos y actividades más dedicadas a sí, a los suyos y a lo demás.

\section{BIBLIOGRAFIA}

BOULIN, J-Y. (1992) "L'Organisation sociale du temps". Futuribles, mayo-junio 1992: 227-237.

BOULIN, J.-Y. (1997) "From working time to city time: the case for a single approach to time policies". Transfer, 4/97: 723-736.

CASTELLS, M. (1997) La era de la información. Vol. 1. Madrid: Alianza.

DURAND, J-P. (2001) "Travail informationnel et flux tendu", en Temps de travail et temps libre, Claude Durand y Alain Pichon (ed.). Bruselas: De Boeck.

FOUCAULT, M., (1975) Surveiller et punir. París: Gallimard.

GORZ, A. (1995) Metamorfosis del trabajo. Madrid: Sistema.

GORZ, A. (1997) "Salir de la sociedad salarial", en El paro y el empleo: enfoques alternativos, Albert Recio, Claus Offe y André Gorz (ed.). Alzira: Germània. GORZ, A. (1999) Miserias del presente, riqueza de lo posible. Barcelona: Paidós.

HARVEY, M. (1999a) Temporalités et construction de l'emploi. Informe para el proyecto TSER La construction sociale de l'emploi. IV Programa marco europeo de investigación. Contrato: ERB SOE 2 CT 973041.

HARVEY, M. (1999b) "Economies of Time: A Framework for Analysing the Restructuring of Employment Relations", en Global Trends in Flexible Labour, Alan Felstead y: Nick Jewson (ed.). Londres: Macmillan.

HÖRNING, L. H.; GERHARD, A.; MICHAILOW, M. (1995) Time Pioneers. Flexible Working Time and New Lifestyles. Cambridge (R.U.): Polity Press. 
KERN, H.; SCHUMANN, M. (1989) El fin de la división del trabajo. Racionalización de la producción industrial. Madrid: Ministerio de Trabajo.

LALLEMENT, M. (2003). Temps, travail et modes de vie. París: PUF.

MARTÍN ARTILES, A. (1995) Flexibilidad y relaciones laborales. Estrategias empresariales $y$ acción sindical. Madrid: CES.

NIELSEN, K. (1991) "Towards Flexible Future - Theories and Politics", en The Politics of Flexibility. Restructuring State and Industry in Britain, Germany and Scandinavia, Bob Jessop, Hans Kastendiek, Klaus Nielsen, Ove K. Pedersen (ed.). Aldershot: Edward Elgar.

PIORE, M.; SABEL, CH. (1990) La segunda ruptura industrial. Madrid: Alianza.

QUIT (TORNS, T.; MIGUÉLEZ, F.; BORRÁS, C.; VERD, J.M.; SÁEZ, L.; ANTENTAS, J.M.; NADAL, M.) (2000) Temps i ciutat. L'estudi del temps a la ciutat més enllà de la seva dimensió horària. Barcelona: CESB.

RECIO, A. (1997a) Trabajo, personas, mercados. Manual de economía laboral. Barcelona/Madrid: Icaria/FUHEM.

RECIO, A. (1997b) "Reducción de la jornada laboral y empleo: interrogantes en torno a una consigna ampliamente popularizada", en Quien parte y reparte... El debate sobre la reducción del tiempo de trabajo, Jorge Riechhman y Albert Recio (ed.). Barcelona: Icaria.

RUESGA, S. M. (dir.); LASIERRA, J. M.; MURAYAMA, C. (2002) Economía del trabajo y política laboral. Madrid: Pirámide.

SIVADON, P.; FERNANDEZ-ZOÏLA, A. (1983) Temps de travail. Temps de viure. Bruselas: Pierre Mardaga.

SUE, R. (1982) Vers une societé du temps libre? París: PUF.

SUE, R. (1994) "Tra i lavoro ed il tempo libero: lemergenza di un tempo di utilità sociale". Sociologia del lavoro, 56: 63-77.

TORNS, T. (1998). "Acerca del reparto del trabajo... y de cómo sacarle provecho". T.E., 190: 14-15.

ZOLL, R. (1988) "Destruction ou réappropiation du temps", en Les temps sociaux, D. Mercure y A. Wallemacq (ed.). París: De Boeck.

ZOLL, R. (1994) "Un nuovo modello di redistribuzione del tempo". Sociologia del lavoro, 56: 88-100.

6. En la misma dirección abunda Lallement (2003) que señala otras antinomias para una situación como la actual a partir de dos tipos de racionalidad en la que se inscribe o des-inscribe el trabajo y a partir de la consideración de las funciones del trabajo en tanto que institución. En las sociedades modernas el trabajo ha contribuido a la integración social de los individuos, al lado de otras instituciones como la religión, familia, escuela. Trabajar ha significado o ha tenido como funciones (y las tiene) implicarse en relaciones sociales múltiples, de cooperación, subordinación, contestación.., a amoldarse a reglas que dan homogeneidad en el tratamiento de necesidades, (remuneración y sus modos, condiciones de trabajo y a compartir creencias comunes y fundamentar identidades colectivas de oficio, de empresa. 